\title{
Precision Measurement of the Boron-to-Carbon Flux Ratio in Cosmic Rays with the Alpha Magnetic Spectrometer on the ISS
}

\author{
Alberto Oliva ${ }^{* \dagger}$ on behalf of the AMS-02 Collaboration \\ Centro de Investigaciones Energéticas, Medioambientales y Tecnológicas - CIEMAT, E-28040 \\ Madrid, Spain
}

\begin{abstract}
The Alpha Magnetic Spectrometer is a high energy particle physics experiment in space. It is taking data continuously since May 2011 on the International Space Station. During the first 5 years of data taking AMS collected 2.3 million boron and 8.3 million carbon nuclei, measuring the $\mathrm{B} / \mathrm{C}$ cosmic rays flux ratio between $1.9 \mathrm{GV}$ to $2.6 \mathrm{TV}$ with an unprecedented accuracy. The $\mathrm{B} / \mathrm{C}$ does not show any significant structure and is well described by a single power-law $R^{\Delta}$ with index $\Delta=-0.333 \pm 0.015$ above $65 \mathrm{GV}$, in good agreement with the asymptotic behaviour expected from cosmic rays diffusion based on Kolmogorov turbulence theory.
\end{abstract}

35th International Cosmic Ray Conference - ICRC2017

10-20 July, 2017

Bexco, Busan, Korea

\footnotetext{
${ }^{*}$ Speaker.

${ }^{\dagger}$ E-mail: alberto.oliva@cern.ch
} 


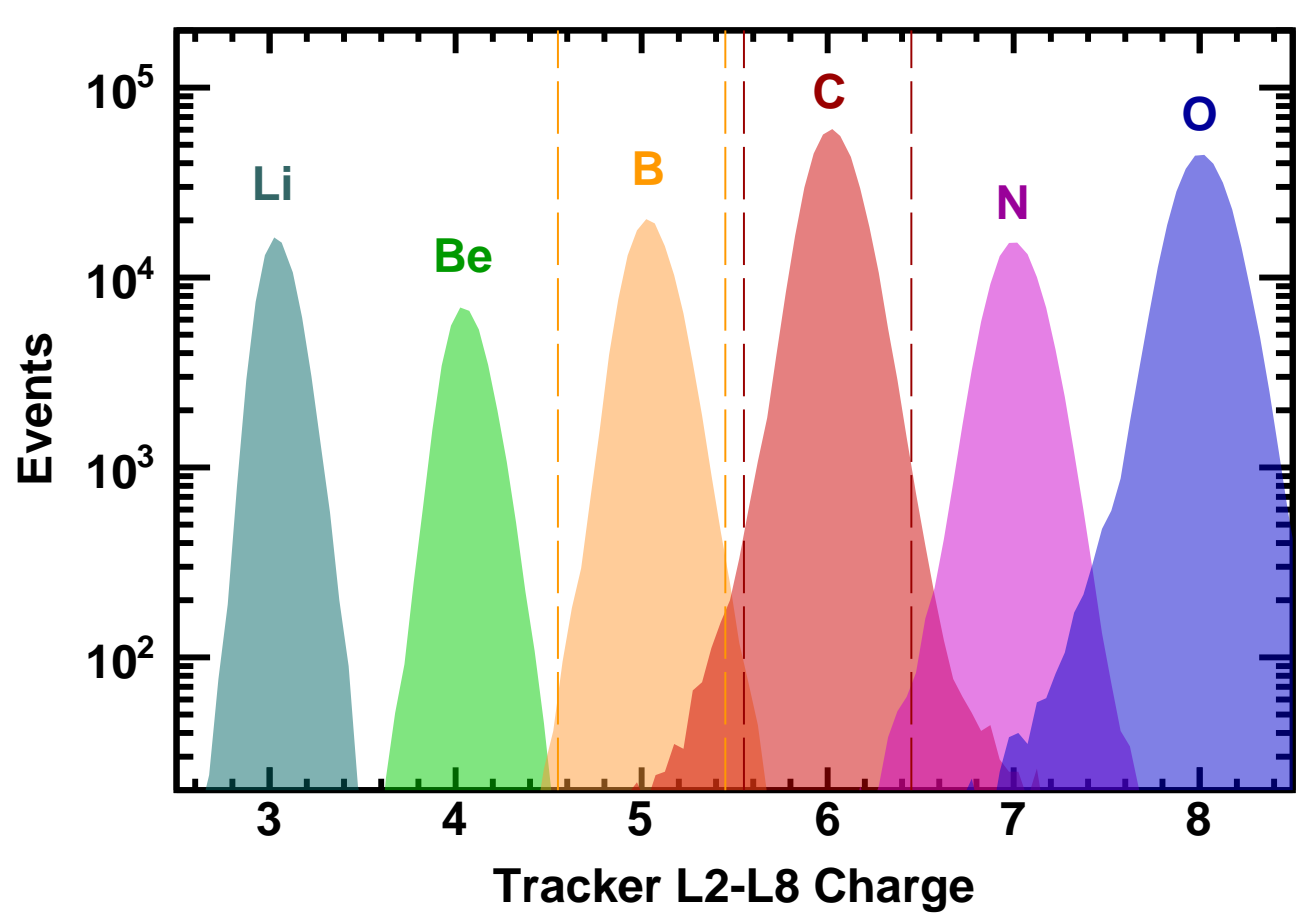

Figure 1: Charge distribution of inner (L2-L8) tracker for samples from $Z=3$ to $Z=8$ in the rigidity range from $4 \mathrm{GV}$ to $10 \mathrm{GV}$, selected by charge on tracker L1, upper TOF and lower TOF. The vertical dashed lines correspond to the charge selection in the inner tracker for boron (orange) and carbon (red).

Cosmic rays carbon nuclei are thought to be mainly produced and accelerated in astrophysical sources, while boron nuclei are produced by the collision of heavier nuclei, such as carbon and oxygen, with the interstellar matter. Therefore, the boron to carbon flux ratio $(\mathrm{B} / \mathrm{C})$ measures the average amount of interstellar material traversed by cosmic rays and is an important observable for the understanding of origin and propagation of cosmic rays [1].

The Alpha Magnetic Spectrometer (AMS) is a high-energy spectrometer in space taking data since May 2011 onboard of the International Space Station. The key AMS [2] parts used in this measurement are the permanent magnet [3], the silicon tracker [4], four planes of time of flight (TOF) scintillation counters [5], and an array of 16 anticoincidence counters. AMS also contains a transition radiation detector (TRD), a ring imaging Čerenkov detector (RICH), and an electromagnetic calorimeter (ECAL).

The particle rigidity $R$ (momentum/charge) is derived by the measurement of the particle curvature in the magnetic field using the $3 \mathrm{~m}$ lever arm of the silicon tracker.

Particle charge $Z$ is measured several times along the particle trajectory: on top of AMS by a layer of Silicon sensors constituting the Tracker L1; in the Inner Tracker by the combination of 7 single layer Tracker measurements (from L2 to L8); in the Upper TOF (UTOF) by the combination of the measurements of two layers of scintillating counters; on the Lower TOF (LTOF) similarly to UTOF; and on the Tracker L9. 
Monte Carlo simulated events were produced using a dedicated program developed by the collaboration based on the GEANT-4.10.1 package [6]. The Glauber-Gribov model was used for the description of the inelastic cross sections. The INCL++ package [7] was used to model nucleusnucleus inelastic interactions below $5 \mathrm{GeV} / \mathrm{n}$ and the DPMJET-II.5 package [8] was used at higher energies. The nucleus-nucleus elastic interaction were modelled using the methods developed for helium nuclei [9].

This analysis uses data collected in the first 5 years of AMS operations. The collection time includes only those seconds during which the detector was in normal operating conditions and, in addition, AMS was pointing within $40^{\circ}$ of the local zenith, and the ISS was outside of the South Atlantic Anomaly. Due to the influence of the geomagnetic field, this collection time for galactic cosmic rays increases with rigidity becoming constant at $1.23 \times 10^{8}$ seconds above $30 \mathrm{GV}$.

Events are required to be downward going and to have a reconstructed track in the inner tracker and passing through the L1. In the highest rigidity region, $R \geq 0.88 \mathrm{TV}$, the track is also required to pass through L9. Track fitting quality criteria such as a $\chi^{2} /$ d.f. $<10$ in the bending coordinate are applied, similar to Ref. [13, 9].

The measured rigidity is required to be greater than a factor of 1.2 times the maximum geomagnetic cutoff within the AMS field of view. The cutoff was calculated by backtracing [11] particles from the top of AMS out to 50 Earth's radii using the most recent IGRF [12] geomagnetic model.

Charge measurements on tracker L1, inner tracker, upper TOF, lower TOF, and, for $R>0.88$ $\mathrm{TV}$, tracker L9 are required to be compatible with charge $Z=5$ for boron, and $Z=6$ for carbon, as shown in Fig. 1 for the inner tracker. This selection yields purities of $90 \%$ to $95 \%$ depending on rigidity for boron, and $99 \%$ for carbon.

The residual background to boron and carbon events resulting from interactions of heavy nuclei such as carbon, nitrogen and oxygen in the material between L1 and L2 (TRD and upper TOF) is evaluated by fitting the charge distribution of tracker L1 with charge distribution templates of B,C,N and O, as shown in Fig. 2. The charge distributions templates are obtained from a selection of non-interacting samples on L2 by the use of the charge measurement with L1 and L3-L8. This residual background is $<3 \%$ for boron sample and $<0.5 \%$ for carbon.

The background from carbon, nitrogen and oxygen interactions on materials above L1 has been estimated from simulation, using MC samples generated according to AMS flux measurements. The simulation of nuclear interactions has been validated using fragmenting events between L1 and inner Tracker [10]. The background from interactions above L1 in the boron sample is $2 \%$ at $2 \mathrm{GV}$ and increases up to $8 \%$ at $2.6 \mathrm{TV}$, while for the carbon sample is $<0.5 \%$ in all rigidity range.

After background subtraction the sample contains $2.3 \times 10^{6}$ boron and $8.3 \times 10^{6}$ carbon nuclei.

The isotropic flux $\Phi_{i}^{Z}$ for nuclei of charge $Z$ in the $i^{\text {th }}$ rigidity bin $\left(R_{i}, R_{i}+\Delta R_{i}\right)$ is given by

$$
\Phi_{i}^{Z}=\frac{N_{i}^{Z}}{A_{i}^{Z} \varepsilon_{i}^{Z} T_{i} \Delta R_{i}}
$$

where $N_{i}^{Z}$ is the number of events of charge $Z$ corrected for bin-to-bin migrations, $A_{i}^{Z}$ is the effective acceptance, $\varepsilon_{i}^{Z}$ is the trigger efficiency, and $T_{i}$ is the collection time. The $\mathrm{B} / \mathrm{C}$ in each rigidity bin 


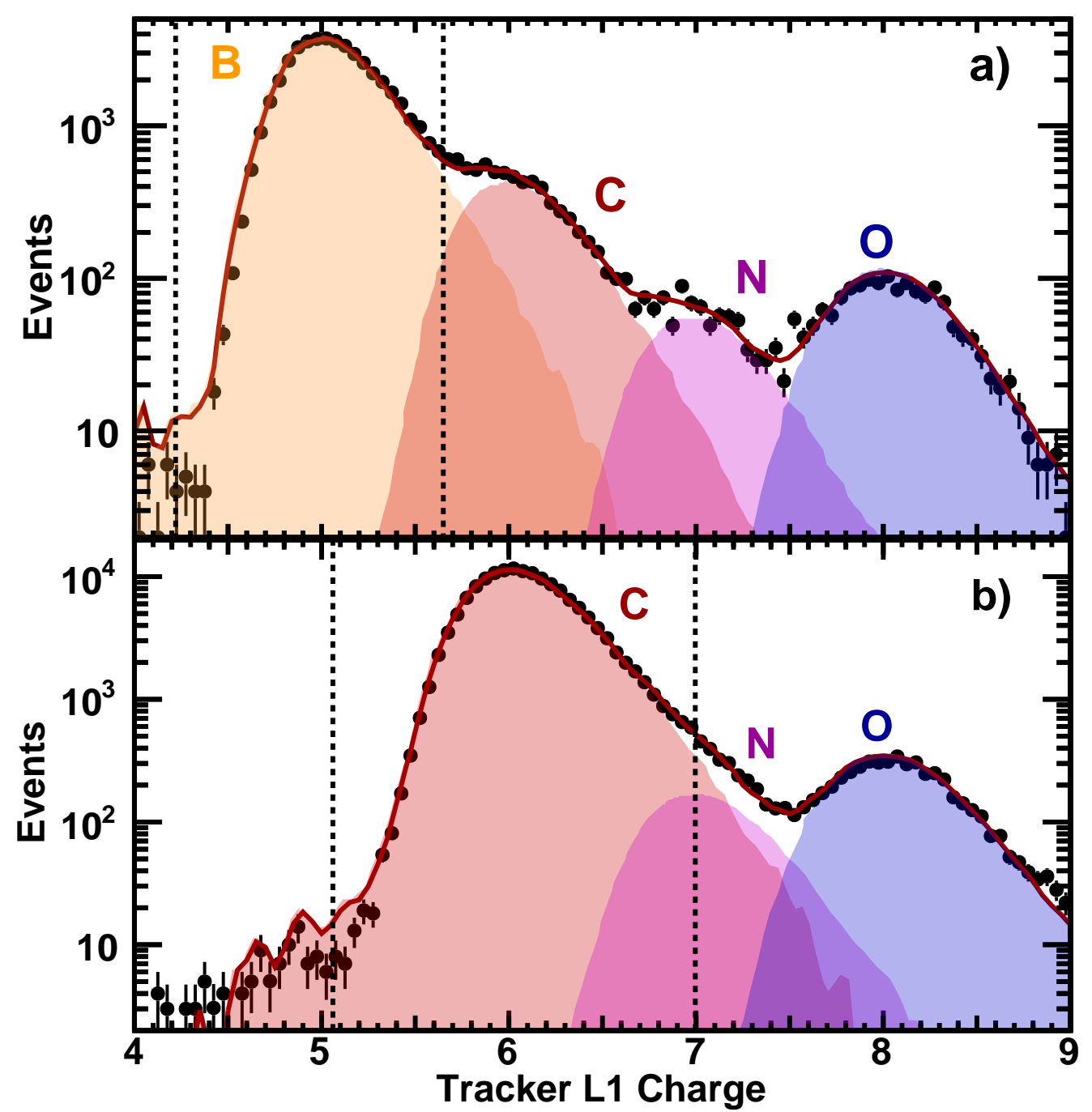

Figure 2: Charge distributions measured by tracker L1 for a) boron and b) carbon events selected by inner tracker in the rigidity range between 9 and $11 \mathrm{GV}$ (dots). The solid red lines show the fit of the charge distribution templates B,C,N and $\mathrm{O}$, to the data. The templates are obtained from a selection of non-interacting samples on L2 by the use of the charge measurement with L1 and L3-L8. The charge selections applied on tracker L1 are shown as vertical dashed lines. The residual backgrounds to the boron and carbon samples are calculated by integrating the charge templates distribution in the selection range,

is then given by

$$
\left(\frac{\mathrm{B}}{\mathrm{C}}\right)_{i}=\frac{\Phi_{i}^{\mathrm{B}}}{\Phi_{i}^{\mathrm{C}}}=\frac{N_{i}^{\mathrm{B}}}{N_{i}^{\mathrm{C}}} \cdot\left[\frac{A_{i}^{\mathrm{B}}}{A_{i}^{\mathrm{C}}} \cdot \frac{\varepsilon_{i}^{\mathrm{B}}}{\varepsilon_{i}^{\mathrm{C}}}\right]^{-1}
$$

The bin-to-bin migration of events was corrected using the unfolding procedure described in Ref. [13] independently for the boron and the carbon samples.

Extensive studies were made of the systematic errors. These errors include the uncertainties in the two background estimations discussed above, in the trigger efficiency, in the acceptance calculation, in the rigidity resolution function and in the absolute rigidity scale [10]. 


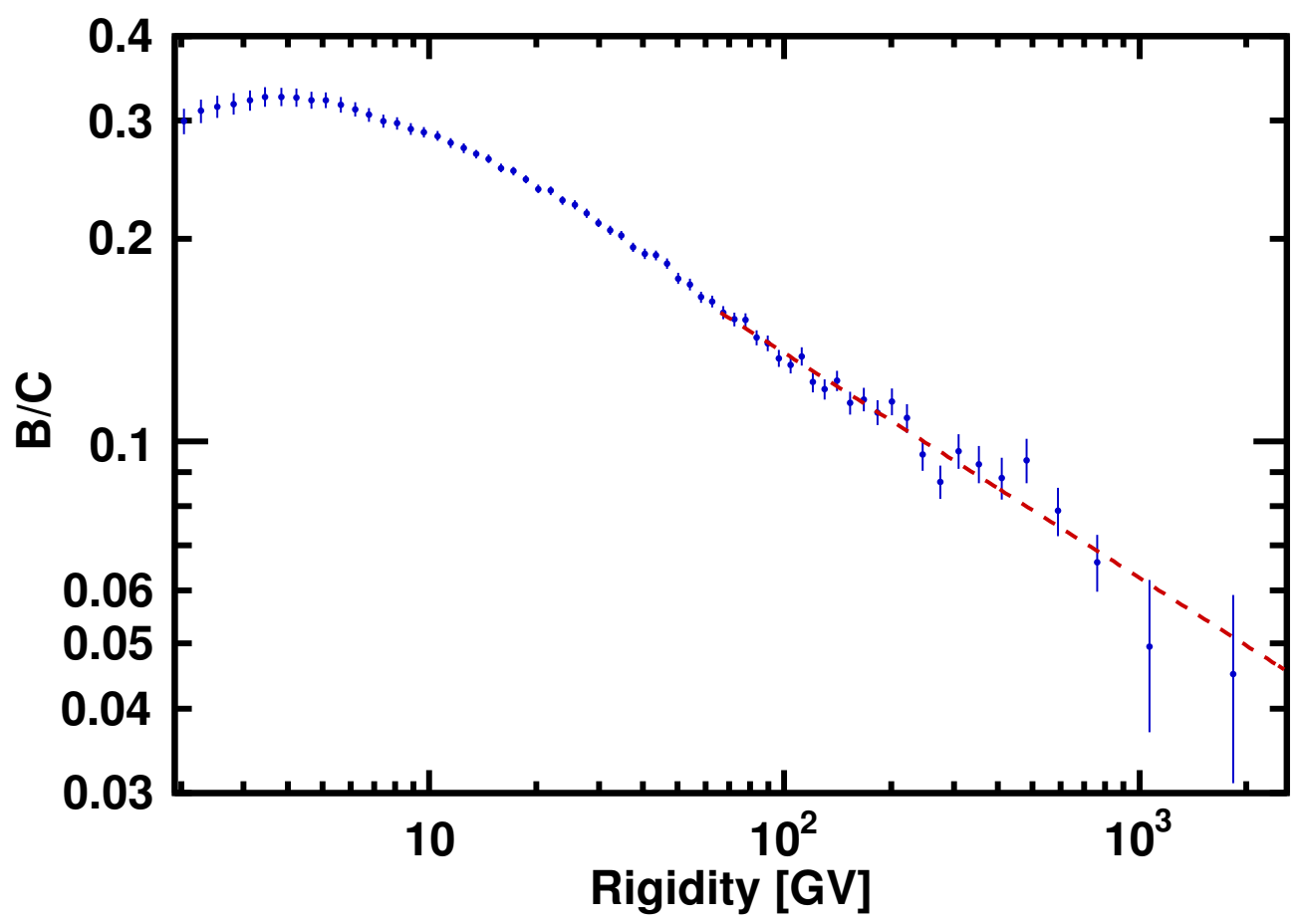

Figure 3: The AMS boron to carbon ratio as a function of rigidity in the interval from $1.9 \mathrm{GV}$ to $2.6 \mathrm{TV}$ based on 2.3 million boron and 8.3 million carbon nuclei. The dashed line shows the single power law fit starting from $65 \mathrm{GV}$ with index $\Delta=-0.333 \pm 0.014$ (fit) \pm 0.005 (sys).

The uncertainty on the acceptance evaluation, due to the uncertainty associated to the inelastic cross-section models, is the dominating systematic error at low energy (is 3\% at $4 \mathrm{GV}$ decreasing to $<2 \%$ above $10 \mathrm{GV}$ ). At the highest energies the unfolding errors, derived from the uncertainties associated to the Tracker rigidity response, dominates the systematic error (less than $1 \%$ below 100 $\mathrm{GV}$ and increasing to $5 \%$ at $2.6 \mathrm{TV}$ ). The statistical fluctuations dominates the overall error above $100 \mathrm{GV}$.

Figure 3 shows the $\mathrm{B} / \mathrm{C}$ as a function of rigidity with the total errors, the sum in quadrature of statistical and systematic errors.

The $\mathrm{B} / \mathrm{C}$ increases with rigidity reaching a maximum at $4 \mathrm{GV}$ then decreases. The $\mathrm{B} / \mathrm{C}$ does not show any significant structures at high energy. Above $65 \mathrm{GV}$ the $\mathrm{B} / \mathrm{C}$ measured by AMS is well fit with a single power law $\mathrm{B} / \mathrm{C}=k R^{\Delta}$ where $k$ is a constant normalisation factor, with a $\chi^{2} / d . f$. $=$ $20 / 24$ and a spectral index $\Delta=-0.333 \pm 0.014$ (fit) \pm 0.005 (sys). The first error (fit) takes into account the statistical and uncorrelated systematic errors, the second (sys) is the error from the remaining systematic errors, namely from the rigidity resolution function, rigidity scale and boron and carbon inelastic cross sections, with proper accounting of the bin-to-bin correlations. We obtain a consistent value of $\Delta$ starting the fitting in the range from 50 to $80 \mathrm{GV}$. The measured $\Delta$ is in good agreement with the Kolmogorov theory of turbulence which predicts $\Delta=-1 / 3$ asymptotically [1].

To compare AMS results with previous measurements a procedure to convert the $\mathrm{B} / \mathrm{C}$ from 


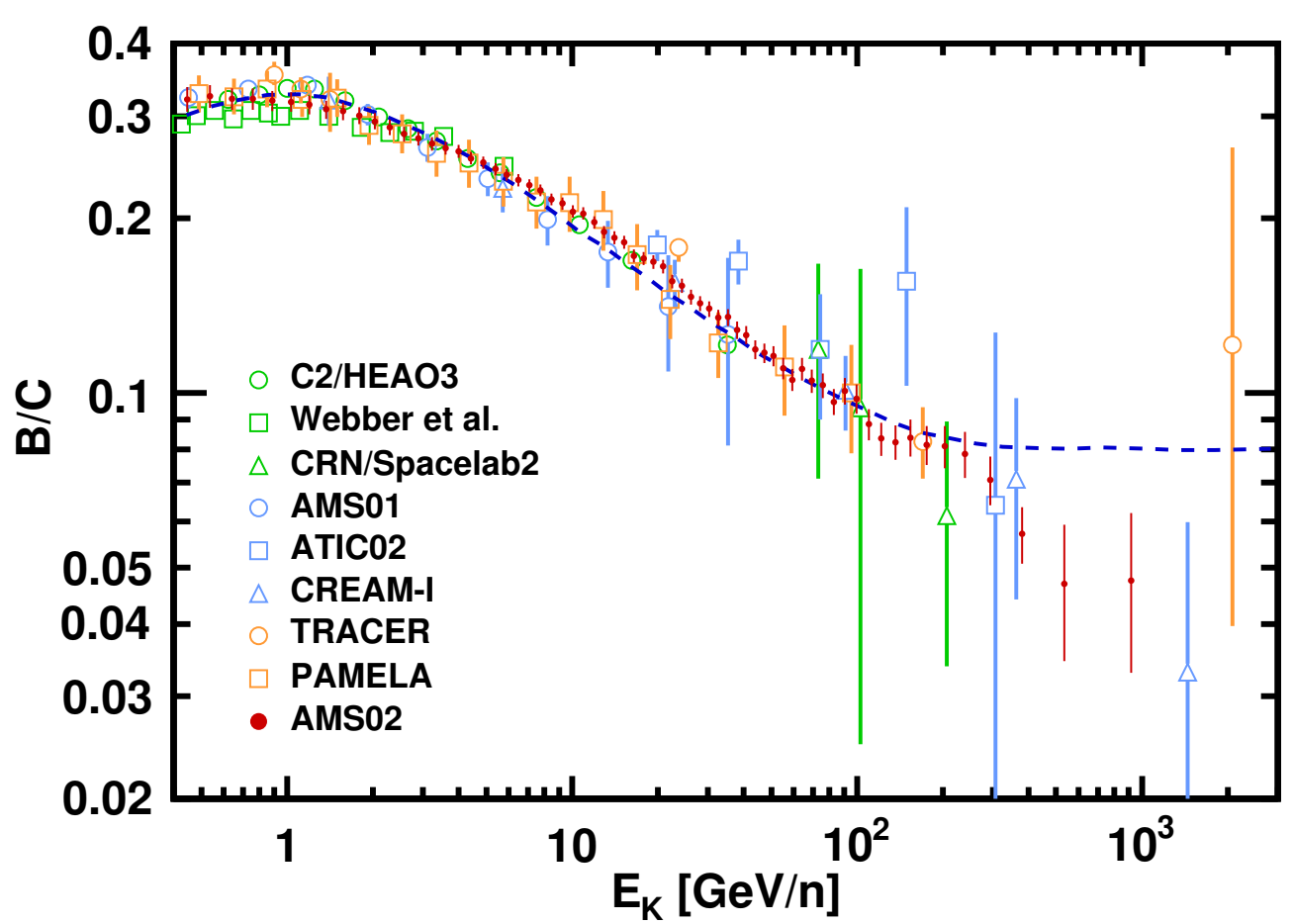

Figure 4: The boron to carbon ratio as a function of kinetic energy per nucleon $E_{K}$ compared with measurements since the year 1980 [14]. The dashed line is the B/C required for the model of Ref. [15].

rigidity to kinetic energy per nucleon $E_{K}$ is used [10]. An additional systematic error due to the conversion procedure is derived varying the boron isotopic composition in the range $Y_{\mathrm{B}}=0.7 \pm 0.1$. Figure 4 shows the AMS B/C together with recent results. Also shown is the $\mathrm{B} / \mathrm{C}$ prediction from an important theoretical model [15], which explains the AMS positron fraction [16] by secondary production in cosmic ray propagation. This is an example of a class of models ruled out by this measurement.

In conclusion, the precise measurement of the boron to carbon flux ratio $\mathrm{B} / \mathrm{C}$ in cosmic rays with rigidity from $1.9 \mathrm{GV}$ to $2.6 \mathrm{TV}$ based on 2.3 million boron and 8.3 million carbon nuclei is presented. The $\mathrm{B} / \mathrm{C}$ increases with rigidity reaching a maximum at $4 \mathrm{GV}$ then decreases. The $\mathrm{B} / \mathrm{C}$ does not show any significant structures. Above $65 \mathrm{GV}$ the $\mathrm{B} / \mathrm{C}$ can be described by a single power law of $\Delta=-0.333 \pm 0.014$ (fit) \pm 0.005 (sys), in good agreement with the Kolmogorov theory of turbulence which predicts $\Delta=-1 / 3$ asymptotically [1].

\section{References}

[1] A. W. Strong, I. V. Moskalenko, and V. S. Ptuskin, Annu. Rev. Nucl. Part. Sci. 57, 285 (2007); A. Castellina and F. Donato, Astrop. Phys. 24, 1-2 (2005).

[2] A. Kounine, Int. J. Mod. Phys. E 211230005 (2012).

[3] K. Lübelsmeyer et al., Nucl. Instrum. Methods Phys. Res., Sect. A 654, 639 (2011). 
[4] B. Alpat et al., Nucl. Instrum. Methods Phys. Res., Sect. A 613, 207 (2010).

[5] V. Bindi et al., Nucl. Instrum. Methods Phys. Res., Sect. A 743, 22 (2014).

[6] J. Allison et al., IEEE Trans. Nucl. Sci. 53, 270 (2006); S. Agostinelli et al., Nucl. Instrum. Methods Phys. Res., Sect. A 506, 250 (2003).

[7] A. Boudard et al., Phys. Rev. C 87014606 (2013); S. Leray et al., J. Phys.: Conf. Series 420, 012065 (2013).

[8] J. Ranft, Phys. Rev. D 51, 64 (1995).

[9] M. Aguilar et al., Phys. Rev. Lett. 115, 211101 (2015).

[10] M. Aguilar et al., Phys. Rev. Lett. 117, 231102 (2016).

[11] J. Alcaraz et al., Phys. Lett. B 484, 10 (2000).

[12] C.C. Finlay et al., Geophys. J. Int. 183, 1216 (2010); E. Thébault et al., Earth Planets Space 67, 79 (2015).

[13] M. Aguilar et al., Phys. Rev. Lett. 114, 171103 (2015).

[14] C.D. Orth et al., Astrophys. J. 226, 1147 (1978); R. Dwyer and P. Meyer, Astrophys. J. 322, 981 (1987); M. Simon et al., Astrophys. J. 239, 712 (1980); J.J. Engelmann et al., Astron. Astrophys. 233, 96 (1990); W. R. Webber et al., Proceedings of 19th Intern. Cosmic Ray Conf., La Jolla, 2, 16 (1985); D. Muller et al., Astrophys. J. 374, 356 (1991); J. Buckley et al., Astrophys. J. 429, 736 (1994); M. Aguilar et al., Astrophys. J. 724, 328 (2010); A.D. Panov et al., Proceedings of the 23rd Intern. Cosmic Ray Conf. Mérida, 2, 3 (2008); H.S. Ahn et al., Astropart. Phys. 30, 133 (2008); A. Obermeier et al., Astrophys. J. 752, 69 (2012); O. Adriani et al., Astrophys. J. 791, 93 (2014).

[15] R. Cowsik, B. Burch and T. Madziwa-Nussinov, Astrophys. J. 786, 124 (2014).

[16] L. Accardo et al., Phys. Rev. Lett. 113, 121101 (2014); 